\title{
Advances in Biological Functions and Clinical Studies of FGF2I
}

\author{
Wei Lin' \\ Tianlei Zhang' \\ Yiyang Zhou ${ }^{2}$ \\ Jinyu Zheng ${ }^{2}$ \\ Zhenlang Lin (D) \\ 'Department of Pediatrics, The Second \\ Affiliated Hospital and Yuying Children's \\ Hospital of Wenzhou Medical University, \\ Wenzhou, 325000, Zhejiang, People's \\ Republic of China; ${ }^{2}$ Wenzhou Medical \\ University, Wenzhou, 325000, Zhejiang, \\ People's Republic of China
}

\begin{abstract}
Fibroblast growth factor 21 (FGF21) regulates many crucial biological processes in human and mammals, particularly metabolic modulation and protective effect after injury. Therefore, determining complex regulatory mechanisms and elucidating the signaling pathway may greatly promote the prevention, diagnosis, and treatment of related injury and metabolic diseases. This review focused on the metabolic modulation and protective effect of FGF21 and summarized the molecular mechanisms and clinical research developments.
\end{abstract}

Keywords: fibroblast growth factor 21, molecular mechanisms, metabolic modulation, protective effect, clinical application

\section{Introduction}

Fibroblast growth factors (FGFs) are widely expressed in the human body and have numerous and complicated physiological functions. FGFs regulate cell growth, proliferation, differentiation, and metabolic modulation, tissue repair, and inflammatory response. ${ }^{1,2}$ FGFs can be divided into three types (classic, intracellular and hormonelike types) based on their action modes. ${ }^{3}$ Classic and intracellular FGFs mostly act in an autocrine or paracrine way, ${ }^{4}$ while hormone-like FGFs mainly act in the endocrine way. ${ }^{5}$ Most autocrine and paracrine FGFs have a high affinity for the heparin glucosamine sulfate (HSGAG), which promotes the binding of the FGFs to the tyrosine kinase FGF receptor (FGFR1-4) on the cell surface, inducing FGFR activation, dimerization, and activation of downstream signaling pathways. ${ }^{6-9}$ HSGAGs can also stabilize FGFs, prevent FGFs degradation, and limit the diffusion range of FGFs. ${ }^{10}$

FGF21 is a member of the FGF19 subfamily in the FGFs family and belongs to the hormone-like FGFs. Compared to other FGFs members, FGF21 has three special features: 1 . It has no obvious mitogenic or tumor-promoting function; ${ }^{11} 2$. The spatial configuration variation of the region that binds to HSGAGs makes it have a weaker affinity for HSGAG, thus not easily bound in the extracellular matrix. However, it enters the circulation in the form of endocrine hormones to regulate metabolism and cross the blood-brain barrier through simple diffusion; ${ }^{12} 3$. It does not directly bind to cell receptors, and it needs $\beta$-Klotho protein to form a stable combination to play its physiological role. Herein, the latest research progress on FGF21 is summarized.

\section{Receptor Characteristics of FGF2 I}

Unlike the classical FGFs, FGF21 binds to FGF receptors (FGFRs) and the coreceptor protein $\beta$-Klotho (a single transmembrane protein expressed during the differentiation of adipose precursor cells into adipocytes). Besides, FGF21 activates 
the signal transduction downstream only when FGFRs and $\beta$-Klotho are dimerized and autophosphorylated. ${ }^{13,14}$ Neither $\beta$-Klotho nor FGFRs can be activated by FGF21 alone. ${ }^{15}$ In the FGF21-FGFRs- $\beta$-Klotho complex, $\beta$-Klotho, a "zip code"-like receptor, is the main high-affinity receptor of FGF21, providing the targeted signals for FGF21. At the same time, FGFR acts as a catalytic subunit that mediates receptor dimerization and intracellular signal transduction. ${ }^{16}$ Studies have also shown that the C-terminus and N-terminus are involved in activating FGF21 receptors, and any deletion of the terminus greatly reduces FGF21 activity. The C-terminus of FGF21 has a sugar-mimicking Ser-pro-Ser motif, which can be recognized and bound by $\beta$-Klotho. Besides, the C-terminus mutation of FGF21 is associated with an affinity decrease of $\beta$-Klotho, indicating that the C-terminal also mediates the binding of FGF21 and $\beta$ Klotho. Although the N-terminal mutation of FGF21 is also associated with decreased activity, it can still bind to $\beta$ Klotho, suggesting that the N-terminal only participates in the receptor activation process. ${ }^{16-19}$ Therefore, complete $\mathrm{C}$-terminal and $\mathrm{N}$-terminal structure and simultaneous binding to $\beta$-Klotho and FGFRs promote the physiological functions of FGF21.

\section{Regulation of FGF2 I Expression Level}

Studies have shown that FGF21 is highly expressed in the liver, skeletal muscle, kidney, heart, fat, and blood vessels. ${ }^{20}$ FGF21 (mainly produced by liver cells) is also expressed in the blood circulation due to its hormone-like characteristics, ${ }^{21}$ targeting the heart, bones, kidneys, small intestine, and brain because of its strong diffusion and distribution ability. ${ }^{13}$ However, FGF21 produced in adipose tissue most acts in an autocrine or paracrine manner on the metabolic regulation of itself and adjacent tissues. ${ }^{22}$ Moreover, the expression level of FGF21 varies greatly in different tissues and under different conditions. FGF21 expression level can be regulated in the following ways;

\section{Regulation by Transcription Factors}

Numerous transcription regulators can also regulate the FGF21 expression level. For instance, peroxisome proliferator-activated receptor (PPAR) can regulate FGF21 expression by binding to the transcriptional regulatory element at the beginning of the FGF21 promoter region, and FGF21 and PPAR can regulate each other. PPAR contains three subtypes: PPAR $\alpha, \operatorname{PPAR} \beta / \delta$, and PPAR $\gamma$.
PPAR $\alpha$ and PPAR $\gamma$ can induce FGF21 expression. PPAR $\alpha$ can induce FGF21 expression in the liver, ${ }^{23}$ and FGF21 level in the liver of mice was significantly increased after the administration of PPAR $\alpha$ agonist fenofibrate, indicating the effect of PPAR $\alpha$ on promoting FGF21 expression. Similarly, PPAR $\gamma$ can regulate the expression and function of FGF21 in adipose tissue. Muise et $\mathrm{al}^{24}$ found that the PPAR $\gamma$ agonist rosiglitazone can activate PPAR $\gamma$, promoting fat cells to produce FGF21 and synergistically regulating FGF21 to accelerate glucose uptake and utilization. Conversely, FGF21 in the tissue can also activate PPAR $\alpha$ and PPAR $\gamma$. Dutchak et $\mathrm{al}^{25}$ indicated that FGF21 induced by PPAR $\gamma$ in adipose tissue can increase its transcription by inhibiting PPAR $\gamma$ ubiquitination. These processes form a cyclic loop of PPAR $\alpha$, PPAR $\gamma$, and FGF21 and feed-forward to regulate FGF21 expression.

\section{Regulation by microRNAs}

Recent studies have indicated that microRNAs (miRNAs) are associated with the regulation of metabolism and metabolic disorders. ${ }^{26}$ MiRNAs primarily bind to the 3'untranslated region (3'UTR) of mRNAs, inhibiting mRNAs translation and regulating the expression of related genes. The activity of the 3'UTR of FGF21 mRNA in the liver can be inhibited by circulating exosomal miRNAs, in which miR-99b is the identified regulatory factor. ${ }^{27}$ Furthermore, anti-miR-577 therapy can protect and restore the insulin regulation of FGF21 and improve the survival and function of diabetic pancreatic $\beta$ cells. $^{28,29}$ MiR-212 can inhibit FGF21 expression in HepG2 cells in non-alcoholic fatty liver disease (NAFLD), while miR-212 inhibitors can increase FGF21 protein level and reduce lipid synthesis. Si-FGF-21 silences the expression of FGF21, significantly increasing the lipid content in HepG2 cells, thus hindering the antilipid effect of miR-212 inhibitors in these cells. ${ }^{30}$ Collectively, these suggest that miR-212 targets FGF21, thus regulating FGF21 expression.

\section{Regulation by Dietary}

Fasting, ketogenic diet, high-sugar diet, and high-fat diet are all important transcription inducers for FGF21 expression. Fasting can strongly induce FGF21 expression. ${ }^{31}$ A ketogenic diet or fasting can significantly increase FGF21 expression by recruiting PPAR $\alpha$ in the region near the FGF21 transcriptional promoter. ${ }^{23,32}$ Notably, neither the ketogenic diet (up to 3 months) nor short- 
term fasting (up to 48 hours) can increase serum FGF21 levels in humans. ${ }^{23}$ However, prolonged fasting (7-10 days) can increase serum FGF21 levels in humans, possibly due to the different metabolic rates and fasting tolerances among different species. ${ }^{33}$ A high-fat diet induces excessive unsaturated fatty acids and bile acids, increasing the transcription and secretion of FGF21 in the liver by activating the farnesoid $\mathrm{X}$ receptor (FXR) and PPAR $\alpha^{34}$ Lundsgaard et $\mathrm{al}^{35}$ found that a high-carbohydrate diet can also induce FGF21 gene expression in the liver.

FGF21 can also be highly expressed under oxidative stress, energy stress, endoplasmic reticulum stress, cold stress, and mitochondrial dysfunction, and its expression is also regulated by sodium butyrate, metformin, glucocorticoids, etc. ${ }^{36-38}$ Animal studies have shown that one-time acute exercise (60 minutes on a treadmill or until repeated electrical stimulation rendered normal running impossible) promotes gene expression in the liver of mice and increases serum levels of FGF21. Clinical trials have also shown that 30 minutes of acute aerobic exercise can also increase circulating FGF21 levels. ${ }^{39}$ FGF21 expression level in skeletal muscle is very low in healthy conditions, and circulating FGF21 mainly comes from the liver. However, the expression of myogenic FGF21 significantly increases in some stress states, such as hunger, endoplasmic reticulum stress, mitochondrial dysfunction, etc. ${ }^{40,41}$ Kim et $\mathrm{al}^{42}$ found that the FGF21 content significantly increased in the soleus muscle of Zucker diabetic obese rats after 12 weeks of resistance exercise. However, the phenomenon was not observed in the gastrocnemius muscle.

\section{The Physiological Function of FGF2 I}

Earlier research on FGF21 function mainly focused on reducing blood sugar and lipids, lowering body weight, improving insulin resistance (IR), and cardiovascular diseases related to abnormal glucose and lipid metabolism, such as coronary heart disease, heart remodeling. ${ }^{12,43}$ In recent years, the functions of FGF21 in inhibiting inflammation, reducing oxidative stress levels, ${ }^{44,45}$ and inhibiting apoptosis of endothelial cells ${ }^{46}$ and cardiomyocytes ${ }^{47}$ have been studied. The regulatory effects of FGF21 on metabolic diseases, such as obesity, hyperlipidemia, and hyperglycemia, the damage repair effects on inflammation and apoptosis, and the blocking effects on some cancers indicate that FGF21 has a broad prospect for development.
Studies have shown that FGF21 mainly participates in the processes of material metabolism, heart and brain damage by activating Adenosine 5'-monophosphate (AMP)activated protein kinase (AMPK), serine/threonine protein kinase (Akt), and extracellular-regulated protein kinases (ERK) and other downstream factors. FGF21 plays metabolic regulation functions, such as promoting glucose uptake, gluconeogenesis, increasing the oxidation of free fatty acids, promoting ketogenesis, increasing energy production and utilization. ${ }^{48,49}$ FGF21 also has the effect of apoptosis protection and neurocognitive function recovery.

\section{FGF2I and Glucose Metabolism}

Insulin, as one of the most important humoral factors regulating glucose metabolism, enhances glucose absorption by activating the Phosphatidylinositol 3-kinase (PI3K)/Akt signaling pathway and promoting the expression of glucose transporter 4 (GLUT4). ${ }^{50}$ FGF21 can also activate the $\mathrm{PI} 3 \mathrm{~K}$ signaling pathway and regulate Akt activation. However, FGF21 mainly inhibits the expression of related gluconeogenesis and glucose production by inducing phosphorylation of atypical protein kinase $\mathrm{C}(\mathrm{PKC}) \mathrm{I} / \mathrm{L}$ and exerts insulin-like hypoglycemic effect to control blood glucose balance. ${ }^{51} \mathrm{Ge}$ et $\mathrm{al}^{52}$ found that glucose transporter-1 (GLUT1) promoter is highly conserved in serum response element (SRE) and E-26 (ETS) binding motifs. FGF21 binds to the receptor to activate the downstream factor extracellular signal-regulated kinases $($ ERK1/2)), and then activate ETS-like protein-1 (ELK-1) and serum response factor (SRF, the downstream target of ERK1/2), enhancing glucose uptake and reducing blood glucose concentration by combining the promoter sequence of GLUT1 gene, trans-activating GLUT1 gene and inducing GLUT1 expression. Furthermore, there can be a synergistic effect between insulin and FGF21. Insulin can activate $\mathrm{PKC}$, enhancing the FGF21 regulation effect on the GLUT1 expression through the ERK1/2-SRF/Elk-1 pathway. ${ }^{53}$ FGF21 can also improve insulin sensitivity and enhance the hypoglycemic effect of insulin. The mammalian target of rapamycin complex 1 (mTORC1) and its downstream effector, ribosomal protein S6 kinase 1 (S6K1), are the key regulators of nutritional overloadinduced insulin resistance and the pathogenesis of type 2 diabetes mellitus (T2DM). ${ }^{54} \mathrm{~S} 6 \mathrm{~K} 1$ phosphorylates insulin receptor substrate 1 (IRS-1), thereby destroying PI3K, a key molecular basis for inducing IR. FGF21 binds to the receptor complex, downregulating mTORC1 in a tuberous sclerosis complex (TSC)-dependent manner, 
thus activating downstream pathways to improve insulin resistance and increase insulin sensitivity. ${ }^{55}$ Notably, FGF21 has a two-way regulatory role in lowering and raising blood sugar levels. Besides, animal experiments and clinical trials have shown different functions of FGF21 and the body regulation mechanism of normal and abnormal metabolism.

Rat experiments showed FGF21 improves IR, increases insulin sensitivity, and reduces blood insulin concentration. ${ }^{12}$ FGF21 analogues also improve IR, reduces blood glucose and insulin levels, and improve glucose tolerance in primates. ${ }^{56}$ Moreover, scholars have discovered that adipose tissue is a key target of FGF21 for glucose metabolism regulation, and a certain amount of adipose tissue in mice is the guarantee for FGF21 to regulate blood sugar. ${ }^{57}$ Clinical trials have shown that FGF21 analogues (LY2405319, PF-05231023, BMS986036, etc.) also lower blood glucose. However, their effect on lowering blood glucose was not significant. ${ }^{17,58,59}$ Further studies have shown that FGF21 also promotes gluconeogenesis and maintains blood glucose balance, preventing hypoglycemia occurrence. $^{12}$

FGF21 reduces blood glucose by promoting the phosphorylation of Akt via insulin, thereby upregulating the number of insulin receptors, increasing insulin sensitivity, and alleviating chronic inflammatory response, thus improving IR, thus regulating glucose metabolism. ${ }^{60-62}$ Second, FGF21 can upregulate adiponectin expression in adipocytes by activating the PPAR $\gamma$ signaling pathway to promote GLUT4 migration from cytoplasm to cell membrane, or directly promoting GLUT1 expression, increasing glucose transport and lowering blood glucose. ${ }^{22,63,64}$ FGF21 can also act on pancreatic islets and indirectly induce a hypoglycemic effect. FGF21 can inhibit islet $\alpha$ cells to reduce glucagon secretion, ${ }^{12}$ inhibiting the synergistic toxicity of glycolipid and cytokine-induced $\beta$ cell apoptosis, reducing the damage of islet $\beta$ cells and enhancing the function of islet $\beta$ cells, thereby improving blood sugar level. ${ }^{65}$

In terms of gluconeogenesis, studies have shown that FGF21 can increase and maintain blood sugar under fasting or starvation. ${ }^{66}$ FGF21 secreted by tissue cells can cross the blood-brain barrier and directly act on the hypothalamus through the hypothalamic-pituitary-adrenal axis, stimulating the synthesis and release of adrenal corticosterone increasing blood glucose via liver gluconeogenesis. ${ }^{67}$ Furthermore, FGF21 can bind to FGFRs in the liver to activate the downstream RAS-RAFMAPK signaling pathway via the $\beta$-Klotho, resulting in a cascade reaction. FGF21 then induces the expression of early liver genes, such as PPAR $\gamma$ coactivator-activated receptor- $\gamma$ coactivator $1 \alpha(\mathrm{PGC} 1 \alpha)$ gene, which increases the transcriptional activity of PPAR $\alpha$. It upregulates the expression of gluconeogenesis-related genes, thereby regulating gluconeogenesis. ${ }^{68,69}$

\section{FGF2I and Lipid Metabolism}

FGF21 is an "adipokine", which regulates blood lipid. It can significantly reduce the plasma levels of total cholesterol (TC), low-density lipoprotein (LDL), and triglyceride (TG) and increase the plasma levels of high-density lipoprotein (HDL) and adiponectin in normal subjects. ${ }^{70}$ A study indicated that diet-induced obese mice and hereditary $\mathrm{db} / \mathrm{db}$ obese mice have weight loss, reversal of liver steatosis, and reduction in plasma TG levels changes after several weeks of continuous FGF21 administration. Plasma TC and LDL levels decrease, while HDL level increases in a dose-dependent manner in mice administered with different FGF21 doses. ${ }^{71,72}$ Similar findings were found in diabetic monkeys. Besides the decrease in blood glucose to the normal level, the decrease of blood insulin level, and the enhancement of the body's sensitivity to insulin, blood lipid also decreased, especially the selective decrease of TG and LDL. At the same time, HDL showed an upward trend after long-term injection of FGF21 in diabetic monkeys. ${ }^{73}$

FGF21 also plays an important role in liver fat metabolism. Liu et $\mathrm{al}^{74}$ indicated that long-term alcohol exposure induces liver damage and steatosis, inducing the body to upregulate FGF21 as compensation. Knockout of the FGF21 gene inhibits $\beta$-oxidation of liver fatty acids, aggravating alcoholic steatohepatitis (ASH). However, recombinant FGF21 alleviates liver steatosis and inflammation induced by ASH. Furthermore, the treatment of rodent and non-human primate model animals of NAFLD, FGF21 analog, and B1344 showed an inhibited liver inflammation and natural immune cell infiltration, reduced liver injury and hepatocyte death, significantly improved liver lipid accumulation, liver fibrosis, and prevented further development of non-alcoholic steatohepatitis (NASH). ${ }^{75}$ However, B1344 has greater potency, higher maximum response, and lower immunogenicity than FGF21. 


\section{FGF2I and Energy Metabolism}

Humans and animals have white adipose tissue (WAT) and brown adipose tissue (BAT). The WAT mainly stores metabolic surplus energy in the form of TG, which causes weight gain. ${ }^{76}$ Unlike WAT, BAT is rich in multi-crystal mitochondria, which is the main source of non-shivering thermogenesis. BAT maintains the body temperature and energy balance by increasing the uptake of glycolipids and using uncoupling protein 1 (UCP1) to decouple biological oxidation from ATP synthesis, thus converting energy released via biological oxidation into heat, reducing ATP synthesis. ${ }^{77,78}$ FGF21 can upregulate the expression of UCP1 and other thermogenesis genes, promoting the "Browning" of WAT, thus regulating the balance between body temperature and energy. ${ }^{79}$ However, scholars have also found that FGF21 regulates energy metabolism only in mice with abnormal metabolism. ${ }^{79,80}$ Furthermore, Liver kinase B1 (LKB1) phosphorylation activates AMPK, thereby increasing the content of NAD+ in cells, activating Sirtuin 1 (SIRT1), thus promoting mitochondrial energy transformation in skeletal muscles. ${ }^{81-83}$ FGF21 in skeletal muscle is also transported to adipose tissue through blood circulation, increasing the level of FGF21 in WAT and further inducing the expression of silent mating type information regulation 2 homolog 1 (SIRT1), PGC1 $\alpha$, UCP1 and other factors, thus promoting the "Browning" of WAT and increasing energy consumption. ${ }^{84}$ Collectively, the above studies indicate that FGF21 maintains the energy balance of the body with abnormal metabolism through the regulation of glucose and lipid metabolism, which does not affect the normal metabolism in the body.

\section{FGF2I and Heart}

Recent studies have shown that FGF21 has an important protective effect on the heart, reflected by reducing myocardial cell apoptosis, antagonism against ischemiareperfusion injury (IRI), and oxidative stress reaction. Roberts et $\mathrm{al}^{85}$ and Cong et $\mathrm{al}^{86}$ showed that FGF21 could inhibit the activity of the apoptotic factor C-caspase 3 through the PI3K /Akt signaling pathway, increase cardiac energy supply through the AMPK pathway, thus inhibiting the apoptosis of cardiomyocytes. Moreover, FGF21 interferes with the PER K-eIF2 $\alpha$-ATF4CHOP signaling axis during endoplasmic reticulum stress, activating the ATF4 signaling pathway and promoting the phosphorylation of c-Jun N-terminal kinase (JNK), thus reducing the accumulation of abnormally folded proteins and inhibiting myocardial cell apoptosis. ${ }^{87}$ FGF21 can also effectively prevent palmitate-induced cardiac apoptosis by upregulating the ERK1/2-dependent p38 mitogenactivated protein kinase (MAPK)-AMPK signaling pathway ${ }^{88}$ and reduce cardiomyocyte IRI and apoptosis after oxidative stress through PI3K/AKT-dependent pathways. 1. FGF21, as an antioxidant factor, induces the expression of antioxidant genes, such as Ucp3, Ucp2, and Sod 2 in the heart, thereby preventing the production of reactive oxygen species (ROS). ${ }^{89} 2$. FGF21 can also activate MAPK to produce a cascade reaction by binding FGFR1c/ $\beta$-klotho. Therefore, FGF21 can promote the activation of AMPK, the target gene of Liver kinase B1 (LKB1), and reduce the accumulation of ROS, inhibiting apoptosis. ${ }^{89}$ 3. FGF21 promotes transcriptional activation of PPAR $\gamma$ by regulating PPAR $\gamma$ coactivator 1 , thus controlling energy metabolism and oxidative stress in multiple tissues, ${ }^{90}$ inducing a protective role on cardiac hypertrophy.

\section{FGF2I and Brain}

Although it is unknown whether the brain can directly generate FGF21, it has been proved that FGF21 can move to the brain through the blood-brain barrier via simple diffusion, ${ }^{91,92}$ playing a key role in metabolic regulation, neuroprotection, and the potential role of cognition.

FGF2 1 can bind to FGFRs and $\beta$-klotho expressed in the hypothalamus, the suprachiasmatic nucleus ( $\mathrm{SCN}$ ), paraventricular nucleus of the hypothalamus (PVN), and other regions of the brain, and act on the Hypothalamus-pituitaryadrenal axis (HPA) and hypothalamic-pituitary-gonad axis (HPG) to regulate the levels of corticotropin-releasing hormone (CRH) and vasopressin (AVP). The increased release of CRH can promote the substantial release of adrenal cortex hormones, which combine with the liver to induce PGC1 $\alpha$ expression. Therefore, FGF21 can upregulate the expression of gluconeogenesis genes, such as glucose 6 phosphatase and phosphoenolpyruvate carboxykinase via PGC1 $\alpha$, increasing liver gluconeogenesis and preventing hypoglycemia. ${ }^{67}$ Moreover, increased CRH in the circulation can increase sympathetic nerve activity in BAT, upregulate UCP1 expression and increase lipolysis in BAT, leading to an increase in energy use and a decrease in weight gain in diet-induced obese mouse models. ${ }^{93}$ The inhibited release of AVP decreases kisspeptin in the hypothalamus, causing 
a decrease in the release of luteinizing hormone, affecting ovulation and fertility. ${ }^{94}$

Several studies have shown that FGF21 has an important protective effect on nerve injury and cognitive impairment. Shahror et al ${ }^{95}$ showed that FGF21 can significantly improve the spatial memory deficits, hippocampal nerve damage, and dendritic morphology abnormalities induced by traumatic brain injuries (TBI). Therefore, FGF21 alleviates TBIinduced defects in neurogenesis and maturation of immature hippocampal neurons, thereby restoring the hippocampal independent learning and memory impairment caused by TBI. Sa-Nguanmoo et $\mathrm{al}^{96}$ also confirmed that FGF21 enhances hippocampal synaptic plasticity, increases the density of dendritic spines, restoring the function of brain mitochondria. FGF21 can prevent defective neuroprotective molecules in astrocytes, alleviating memory dysfunction and neurodegeneration, such as amyloid plaque pathology and pathological Tau hyperphosphorylation, thus acting as a neuroprotective agent in Alzheimer's disease (AD). ${ }^{97,98}$ FGF21 can also protect the blood-brain barrier after ischemic stroke by activating PPAR $\gamma$ in cerebral blood vessels, ${ }^{99}$ antagonize the M1 polarization of microglia and the accumulation of pro-inflammatory cytokines by inhibiting NF- $\mathrm{\kappa B}$ and upregulating PPAR $\gamma$, and reduce cerebral edema and inflammation after TBI and stroke. ${ }^{100,101}$

\section{Clinical Application}

The level of FGF21 in normal human circulation is low, while it is significantly increased in patients with atherosclerosis, hypertension, and liver disease. ${ }^{102-104}$ Some scholars have also indicated that FGF21 levels in obese or hyperlipidemia patients are 1.5-2 times higher than in normal people. Patients with renal insufficiency may also accumulate FGF21, while FGF21 levels in patients with long-term malnutrition (such as patients with anorexia nervosa) are low. ${ }^{105-107}$ FGF21 expression is decreased in patients with type 1 diabetes mellitus (T1DM) and increased in patients with T2DM in individuals with abnormal glucose metabolism, indicating that FGF21 resistance or compensatory increase may exist in patients with T2DM. ${ }^{108}$ Similarly, FGF21 up-regulation occurs in patients with metabolic syndrome, ${ }^{109}$ possibly because FGF21 plays a compensatory role in the early stage of the disease, promoting the occurrence and development of the disease. Li et $\mathrm{al}^{110}$ found that the plasma FGF21 content and liver FGF21 levels are significantly higher in NAFLD patients than in healthy people. Therefore, FGF21 may be a new biomarker for NAFLD diagnosis. Taken together, these findings indicate that FGF21 has broad prospects as a serum marker in the prevention, diagnosis, treatment, and efficacy evaluation of metabolic abnormalities. Presently, FGF21 has been partially used in clinical practice.

The routine auxiliary examination items, such as serum lactic acid, alanine, creatine kinase have low specificity and sensitivity during the diagnosis of mitochondrial diseases. Recent studies have shown that serum levels of FGF21 are elevated when mitochondrial DNA mutations occur in skeletal muscle but not when similar mutations occur in other organs. Therefore, serum FGF21 is a relatively good biomarker for muscle mitochondrial diseases than other indicators. ${ }^{111}$

Serum FG21 can also be used for early disease detection. For instance, the incidence rate of myocardial ischemia, cardiac hypertrophy, and diabetic cardiomyopathy is positively correlated with serum FGF21 levels. ${ }^{112-114}$ Some studies have also indicated that higher serum FGF21 levels are associated with a higher risk of coronary heart disease, NAFLD, and T2DM. ${ }^{89,115-117}$ Besides, higher circulating FGF21 levels are associated with higher mortality in patients with end-stage renal disease. ${ }^{110}$ However, animal experiments and clinical trials have shown different results. Therefore, further studies are necessary to determine whether FGF21 can be used as a molecular marker for the early diagnosis and evaluation of related diseases.

FGF21 also has important clinical significance for disease treatment. Animal experiments have shown that the metabolic phenotypes, such as decreased blood glucose, serum TC and TG levels, increased insulin sensitivity and glucose uptake of two groups of mice overexpressing human and mouse FGF21 are highly similar. However, there are also some differences. For instance, the weight and body fat rate of mice overexpressing human FGF21 decreased, while those overexpressing mouse FGF21 were obese and unresponsive despite having normal metabolism. ${ }^{17}$ This study provides a basis for the clinical treatment of FGF21. Clinically, FGF21 levels are elevated in patients with various metabolic abnormalities. In contrast, the findings have shown that FGF21 effectively treats various metabolic abnormalities in animal models. However, it is speculated that the phenomenon is similar to hyperinsulinemia. The increase of FGF21 may be due to "FGF21 resistance" produced by the activation of its compensatory mechanism, ${ }^{118}$ indicating that the elevated FGF21 is the body's self-protection mechanism. 
Therefore, FGF21 can treat metabolic disorders, such as diabetes and lipid metabolism disorders, because it does not possess the classic mitogenic activity of the FGFs. ${ }^{119}$

\section{Conclusion and Prospect}

This article reviews the metabolic regulation, injury protection, and other physiological and pathological functions of FGF21 in various tissues and organs in the body, such as the liver, heart, brain, and adipose tissue, and related regulatory mechanisms. To date, the relevant signal pathways of FGF21 have not been fully elucidated. Therefore, an in-depth study on the systemic biological functions and regulatory pathways of FGF21 can promote the prevention and treatment of metabolic disorders, such as diabetes and obesity, and cardiac and brain diseases, such as myocardial IRI and AD, TBI, and stroke.

\section{Disclosure}

The authors report no conflicts of interest in this work.

\section{References}

1. Itoh N, Ornitz DM. Evolution of the Fgf and Fgfr gene families. Trends Genet. 2004;20:563-569. doi:10.1016/j.tig.2004.08.007

2. Kelleher FC, O'Sullivan H, Smyth E, McDermott R, Viterbo A. Fibroblast growth factor receptors, developmental corruption and malignant disease. Carcinogenesis. 2013;34:2198-2205. doi:10.1093/carcin/bgt254

3. Fukumoto S. Actions and mode of actions of FGF19 subfamily members. Endocr J. 2008;55:23-31. doi:10.1507/endocrj.KR07E002

4. Mohammadi M, Olsen SK, Goetz R. A protein canyon in the FGF-FGF receptor dimer selects from an a la carte menu of heparan sulfate motifs. Curr Opin Struct Biol. 2005;15:506-516. doi:10.1016/j.sbi.2005.09.002

5. Goetz R, Beenken A, Ibrahimi OA, et al. Molecular insights into the klotho-dependent, endocrine mode of action of fibroblast growth factor 19 subfamily members. Mol Cell Biol. 2007;27:3417-3428. doi:10.1128/MCB.02249-06

6. Itoh N, Ornitz DM. Functional evolutionary history of the mouse Fgf gene family. Dev Dyn. 2008;237:18-27. doi:10.1002/ dvdy. 21388

7. Ornitz DM, Xu J, Colvin JS, et al. Receptor specificity of the fibroblast growth factor family. $J$ Biol Chem. 1996;271:15292-15297. doi:10.1074/jbc.271.25.15292

8. Ornitz DM. FGFs, heparan sulfate and FGFRs: complex interactions essential for development. Bioessays. 2000;22:108-112. doi:10.1002/(SICI)1521-1878(200002)22:2<108::AID-BIES2>3. $0 . \mathrm{CO} ; 2-\mathrm{M}$

9. Mohammadi M, Olsen SK, Ibrahimi OA. Structural basis for fibroblast growth factor receptor activation. Cytokine Growth Factor Rev. 2005;16:107-137. doi:10.1016/j.cytogfr.2005.01.008

10. Hacker U, Nybakken K, Perrimon N. Heparan sulphate proteoglycans: the sweet side of development. Nat Rev Mol Cell Biol. 2005;6:530-541. doi:10.1038/nrm1681

11. Chen R, Ovbiagele B, Feng W. Diabetes and stroke: epidemiology, pathophysiology, pharmaceuticals and outcomes. Am J Med Sci. 2016;351:380-386. doi:10.1016/j.amjms.2016.01.011
12. Kharitonenkov A, Shiyanova TL, Koester A, et al. FGF-21 as a novel metabolic regulator. $J$ Clin Invest. 2005;115:1627-1635. doi:10.1172/JCI23606

13. Kharitonenkov A, DiMarchi R. Fibroblast growth factor 21 night watch: advances and uncertainties in the field. $J$ Intern Med. 2017;281:233-246. doi:10.1111/joim.12580

14. Ogawa Y, Kurosu H, Yamamoto M, et al. BetaKlotho is required for metabolic activity of fibroblast growth factor 21. Proc Natl Acad Sci U S A. 2007;104:7432-7437. doi:10.1073/ pnas.0701600104

15. Hayden MS, West AP, Ghosh S. NF-kB and the immune response. Oncogene. 2006;25:6758-6780. doi:10.1038/sj.onc.1209943

16. Lee S, Choi J, Mohanty J, et al. Structures of $\beta$-klotho reveal a 'zip code'-like mechanism for endocrine FGF signalling. Nature. 2018;553:501-505. doi:10.1038/nature25010

17. Gaich G, Chien JY, Fu H, et al. The effects of LY2405319, an FGF21 analog, in obese human subjects with type 2 diabetes. Cell metab. 2013;18:333-340. doi:10.1016/j.cmet.2013.08.005

18. Veniant MM, Komorowski R, Chen P, et al. Long-acting FGF21 has enhanced efficacy in diet-induced obese mice and in obese rhesus monkeys. Endocrinology. 2012;153:4192-4203. doi:10.1210/en.2012-1211

19. Zhang M, Liu Y, Xiong ZY, Deng ZY, Song HL, An ZM. Changes of plasma fibroblast growth factor-21 (FGF-21) in oral glucose tolerance test and effects of metformin on FGF-21 levels in type 2 diabetes mellitus. Endokrynol Pol. 2013;64:220-224.

20. Xie T, Leung PS. Fibroblast growth factor 21: a regulator of metabolic disease and health span. Am J Physiol Endocrinol Metab. 2017;313:E292-302. doi:10.1152/ajpendo.00101.2017

21. Markan KR, Naber MC, Ameka MK, et al. Circulating FGF21 is liver derived and enhances glucose uptake during refeeding and overfeeding. Diabetes. 2014;63:4057-4063. doi:10.2337/db140595

22. Lin Z, Tian H, Lam KS, et al. Adiponectin mediates the metabolic effects of FGF21 on glucose homeostasis and insulin sensitivity in mice. Cell Metab. 2013;17:779-789.

23. Badman MK, Pissios P, Kennedy AR, Koukos G, Flier JS, Maratos-Flier E. Hepatic fibroblast growth factor 21 is regulated by PPARalpha and is a key mediator of hepatic lipid metabolism in ketotic states. Cell Metab. 2007;5:426-437. doi:10.1016/j. cmet.2007.05.002

24. Muise ES, Azzolina B, Kuo DW, et al. Adipose fibroblast growth factor 21 is up-regulated by peroxisome proliferator-activated receptor gamma and altered metabolic states. Mol Pharmacol. 2008;74:403-412. doi:10.1124/mol.108.044826

25. Dutchak PA, Katafuchi T, Bookout AL, et al. Fibroblast growth factor-21 regulates PPARgamma activity and the antidiabetic actions of thiazolidinediones. Cell. 2012;148:556-567. doi:10.1016/j.cell.2011.11.062

26. Rottiers V, Naar AM. MicroRNAs in metabolism and metabolic disorders. Nat Rev Mol Cell Biol. 2012;13:239-250. doi:10.1038/ nrm3313

27. Thomou T, Mori MA, Dreyfuss JM, et al. Adipose-derived circulating miRNAs regulate gene expression in other tissues. Nature. 2017;542:450-455. doi:10.1038/nature21365

28. Chen XY, Li GM, Dong Q, Peng H. MiR-577 inhibits pancreatic beta-cell function and survival by targeting fibroblast growth factor 21 (FGF-21) in pediatric diabetes. Genet Mol Res. 2015;14:15462-15470. doi:10.4238/2015.November.30.24

29. Agarwal V, Bell GW, Nam JW, Bartel DP. Predicting effective microRNA target sites in mammalian mRNAs. Elife. 2015;4. doi:10.7554/eLife.05005

30. Xiao J, Bei Y, Liu J, et al. miR-212 downregulation contributes to the protective effect of exercise against non-alcoholic fatty liver via targeting FGF-21. J Cell Mol Med. 2016;20:204-216. doi: $10.1111 /$ jcmm. 12733 
31. Fazeli PK, Lun M, Kim SM, et al. FGF21 and the late adaptive response to starvation in humans. $J$ CLIN INVEST. 2015;125:4601-4611. doi:10.1172/JCI83349

32. Inagaki T, Lin VY, Goetz R, Mohammadi M, Mangelsdorf DJ, Kliewer SA. Inhibition of growth hormone signaling by the fasting-induced hormone FGF21. Cell Metab. 2008;8:77-83. doi:10.1016/j.cmet.2008.05.006

33. Galman C, Lundasen T, Kharitonenkov A, et al. The circulating metabolic regulator FGF21 is induced by prolonged fasting and PPARalpha activation in man. CELL METAB. 2008;8:169-174. doi:10.1016/j.cmet.2008.06.014

34. Cyphert HA, Ge X, Kohan AB, Salati LM, Zhang Y, Hillgartner FB. Activation of the farnesoid $X$ receptor induces hepatic expression and secretion of fibroblast growth factor 21 . $J$ Biol Chem. 2012;287:25123-25138. doi:10.1074/jbc. M112.375907

35. Lundsgaard AM, Fritzen AM, Sjoberg KA, et al. Circulating FGF21 in humans is potently induced by short term overfeeding of carbohydrates. Mol Metab. 2017;6:22-29. doi:10.1016/j. molmet.2016.11.001

36. Li H, Gao Z, Zhang J, et al. Sodium butyrate stimulates expression of fibroblast growth factor 21 in liver by inhibition of histone deacetylase 3. Diabetes. 2012;61:797-806. doi:10.2337/db110846

37. Nygaard EB, Vienberg SG, Orskov C, Hansen HS, Andersen B. Metformin stimulates FGF21 expression in primary hepatocytes. Exp Diabetes Res. 2012;2012:465282. doi:10.1155/2012/465282

38. Patel R, Bookout AL, Magomedova L, et al. Glucocorticoids regulate the metabolic hormone FGF21 in a feed-forward loop. MOL ENDOCRINOL. 2015;29:213-223. doi:10.1210/me.20141259

39. Kim KH, Kim SH, Min YK, Yang HM, Lee JB, Lee MS. Acute exercise induces FGF21 expression in mice and in healthy humans. PLoS One. 2013;8:e63517. doi:10.1371/journal. pone. 0063517

40. Keipert S, Ost M, Johann K, et al. Skeletal muscle mitochondrial uncoupling drives endocrine cross-talk through the induction of FGF21 as a myokine. Am J Physiol Endocrinol Metab. 2014;306: E469-82. doi:10.1152/ajpendo.00330.2013

41. Vandanmagsar B, Warfel JD, Wicks SE, et al. Impaired mitochondrial fat oxidation induces FGF21 in muscle. Cell Rep. 2016;15:1686-1699. doi:10.1016/j.celrep.2016.04.057

42. Kim HJ, Song W. Resistance training increases fibroblast growth factor-21 and irisin levels in the skeletal muscle of Zucker diabetic fatty rats. $J$ Exerc Nutrition Biochem. 2017;21:50-54. doi:10.20463/jenb. 2017.0008

43. Redondo-Angulo I, Mas-Stachurska A, Sitges M, et al. Fgf21 is required for cardiac remodeling in pregnancy. Cardiovasc Res. 2017;113:1574-1584. doi:10.1093/cvr/cvx088

44. Yu D, Ye X, Che R, et al. FGF21 exerts comparable pharmacological efficacy with Adalimumab in ameliorating collagen-induced rheumatoid arthritis by regulating systematic inflammatory response. Biomed Pharmacother. 2017;89:751-760. doi:10.1016/j.biopha.2017.02.059

45. Luo Y, Ye S, Chen X, Gong F, Lu W, Li X. Rush to the fire: FGF21 extinguishes metabolic stress, metaflammation and tissue damage. Cytokine Growth Factor Rev. 2017;38:59-65. doi:10.1016/j.cytogfr.2017.08.001

46. Chen A, Liu J, Zhu J, et al. FGF21 attenuates hypoxiainduced dysfunction and apoptosis in HPAECs through alleviating endoplasmic reticulum stress. Int J Mol Med. 2018;42:1684-1694. doi:10.3892/ijmm.2018.3705

47. Wang S, Wang Y, Zhang Z, Liu Q, Gu J. Cardioprotective effects of fibroblast growth factor 21 against doxorubicin-induced toxicity via the SIRT1/LKB1/AMPK pathway. Cell Death Dis. 2017;8:e3018. doi:10.1038/cddis.2017.410
48. Montagner A, Polizzi A, Fouche E, et al. Liver PPAR $\alpha$ is crucial for whole-body fatty acid homeostasis and is protective against NAFLD. Gut. 2016;65:1202-1214. doi:10.1136/gutjnl-2015310798

49. Yu Y, Zhang XH, Ebersole B, Ribnicky D, Wang ZQ. Bitter melon extract attenuating hepatic steatosis may be mediated by FGF21 and AMPK/Sirt1 signaling in mice. Sci Rep. 2013;3:3142. doi:10.1038/srep03142

50. Huang X, Liu G, Guo J, Su Z. The PI3K/AKT pathway in obesity and type 2 diabetes. Int $J$ Biol Sci. 2018;14:1483-1496. doi:10.7150/ijbs.27173

51. Kong LJ, Feng W, Wright M, et al. FGF21 suppresses hepatic glucose production through the activation of atypical protein kinase Ciota/lambda. Eur J Pharmacol. 2013;702:302-308. doi:10.1016/j.ejphar.2012.11.065

52. Ge X, Chen C, Hui X, Wang Y, Lam KS, Xu A. Fibroblast growth factor 21 induces glucose transporter-1 expression through activation of the serum response factor/Ets-like protein-1 in adipocytes. J Biol Chem. 2011;286:34533-34541. doi:10.1074/ jbc.M111.248591

53. Woo YC, Xu A, Wang Y, Lam KS. Fibroblast growth factor 21 as an emerging metabolic regulator: clinical perspectives. Clin Endocrinol. 2013;78:489-496. doi:10.1111/cen.12095

54. Um SH, D'Alessio D, Thomas G. Nutrient overload, insulin resistance, and ribosomal protein S6 kinase 1, S6K1. Cell Metab. 2006;3:393-402. doi:10.1016/j.cmet.2006.05.003

55. Gong Q, Hu Z, Zhang F, et al. Fibroblast growth factor 21 improves hepatic insulin sensitivity by inhibiting mammalian target of rapamycin complex 1 in mice. Hepatology. 2016;64:425-438. doi:10.1002/hep.28523

56. Adams AC, Halstead CA, Hansen BC, et al. LY2405319, an engineered FGF21 variant, improves the metabolic status of diabetic monkeys. PLoS One. 2013;8:e65763. doi:10.1371/journal. pone. 0065763

57. Veniant MM, Hale C, Helmering J, et al. FGF21 promotes metabolic homeostasis via white adipose and leptin in mice. PLoS One. 2012;7:e40164. doi:10.1371/journal.pone.0040164

58. Talukdar S, Zhou Y, Li D, et al. A long-acting FGF21 molecule, PF-05231023, decreases body weight and improves lipid profile in non-human primates and type 2 diabetic subjects. Cell Metab. 2016;23:427-440. doi:10.1016/j.cmet.2016.02.001

59. Charles ED, Neuschwander-Tetri BA, Pablo FJ, et al. Pegbelfermin (BMS-986036), PEGylated FGF21, in patients with obesity and type 2 diabetes: results from a randomized phase 2 study. Obesity. 2019;27:41-49. doi:10.1002/oby.22344

60. Lee DV, Li D, Yan Q, et al. Fibroblast growth factor 21 improves insulin sensitivity and synergizes with insulin in human adipose stem cell-derived (hASC) adipocytes. PLoS One. 2014;9: e111767. doi:10.1371/journal.pone.0111767

61. Yu D, Ye X, Wu Q, et al. Insulin sensitizes FGF21 in glucose and lipid metabolisms via activating common AKT pathway. Endocrine. 2016;52:527-540. doi:10.1007/s12020-015-0801-9

62. Potthoff MJ, Kliewer SA, Mangelsdorf DJ. Endocrine fibroblast growth factors 15/19 and 21: from feast to famine. Genes Dev. 2012;26:312-324. doi:10.1101/gad.184788.111

63. Cho YM, Kim DH, Lee KH, Jeong SW, Kwon OJ. The IRE1alpha-XBP1s pathway promotes insulin-stimulated glucose uptake in adipocytes by increasing PPARgamma activity. Exp Mol Med. 2018;50:1-15. doi:10.1038/s12276-018-0131-0

64. Li K, Li L, Yang M, Liu H, Boden G, Yang G. The effects of fibroblast growth factor-21 knockdown and over-expression on its signaling pathway and glucose-lipid metabolism in vitro. Mol Cell Endocrinol. 2012;348:21-26. doi:10.1016/j.mce.2011.07.026

65. Bell GI, Polonsky KS. Diabetes mellitus and genetically programmed defects in beta-cell function. Nature. 2001;414:788-791. doi:10.1038/414788a 
66. Babaknejad N, Nayeri $H$, Hemmati R, Bahrami $S$, Esmaillzadeh A. An overview of FGF19 and FGF21: the therapeutic role in the treatment of the metabolic disorders and obesity. Horm Metab Res. 2018;50:441-452. doi:10.1055/a-0623-2909

67. Liang Q, Zhong L, Zhang J, et al. FGF21 maintains glucose homeostasis by mediating the cross talk between liver and brain during prolonged fasting. Diabetes. 2014;63:4064-4075.

68. Mai K, Andres J, Biedasek K, et al. Free fatty acids link metabolism and regulation of the insulin-sensitizing fibroblast growth factor-21. Diabetes. 2009;58:1532-1538. doi:10.2337/db08-1775

69. Fisher FM, Estall JL, Adams AC, et al. Integrated regulation of hepatic metabolism by fibroblast growth factor 21 (FGF21) in vivo. Endocrinology. 2011;152:2996-3004. doi:10.1210/en.2011-0281

70. Dong JQ, Rossulek M, Somayaji VR, et al. Pharmacokinetics and pharmacodynamics of PF-05231023, a novel long-acting FGF21 mimetic, in a first-in-human study. $\mathrm{Br} J$ Clin Pharmacol. 2015;80:1051-1063. doi:10.1111/bcp.12676

71. Dushay J, Chui PC, Gopalakrishnan GS, et al. Increased fibroblast growth factor 21 in obesity and nonalcoholic fatty liver disease. Gastroenterology. 2010;139:456-463. doi:10.1053/j. gastro.2010.04.054

72. Li X, Ge H, Weiszmann J, et al. Inhibition of lipolysis may contribute to the acute regulation of plasma FFA and glucose by FGF21 in ob/ob mice. FEBS Lett. 2009;583:3230-3234. doi:10.1016/j.febslet.2009.09.012

73. Kharitonenkov A, Wroblewski VJ, Koester A, et al. The metabolic state of diabetic monkeys is regulated by fibroblast growth factor-21. Endocrinology. 2007;148(2):774-781. doi:10.1210/ en.2006-1168

74. Liu Y, Zhao C, Xiao J, et al. Fibroblast growth factor 21 deficiency exacerbates chronic alcohol-induced hepatic steatosis and injury. Sci Rep. 2016;6:31026. doi:10.1038/srep31026

75. Cui A, Li J, Ji S, et al. The effects of B1344, a novel fibroblast growth factor 21 analog, on nonalcoholic steatohepatitis in nonhuman primates. Diabetes. 2020;69:1611-1623. doi:10.2337/ db20-0209

76. Villarroya F, Cereijo R, Villarroya J, Giralt M. Brown adipose tissue as a secretory organ. Nat Rev Endocrinol. 2017;13:26-35. doi:10.1038/nrendo.2016.136

77. Kwon MM, O'Dwyer SM, Baker RK, Covey SD, Kieffer TJ. FGF21-mediated improvements in glucose clearance require uncoupling protein 1. CELL REP. 2015;13:1521-1527. doi:10.1016/j.celrep.2015.10.021

78. Sidossis L, Kajimura S. Brown and beige fat in humans: thermogenic adipocytes that control energy and glucose homeostasis. J Clin Invest. 2015;125:478-486. doi:10.1172/JCI78362

79. Fisher FM, Kleiner $\mathrm{S}$, Douris $\mathrm{N}$, et al. FGF21 regulates PGC-1alpha and browning of white adipose tissues in adaptive thermogenesis. Genes Dev. 2012;26:271-281. doi:10.1101/ gad.177857.111

80. Veniant MM, Sivits G, Helmering J, et al. Pharmacologic effects of FGF21 are independent of the "Browning" of white adipose tissue. Cell Metab. 2015;21:731-738. doi:10.1016/j. cmet.2015.04.019

81. Chau MD, Gao J, Yang Q, Wu Z, Gromada J. Fibroblast growth factor 21 regulates energy metabolism by activating the AMPK-SIRT1-PGC-1alpha pathway. Proc Natl Acad Sci U S A. 2010;107:12553-12558. doi:10.1073/pnas.1006962107

82. Loyd C, Magrisso IJ, Haas M, et al. Fibroblast growth factor 21 is required for beneficial effects of exercise during chronic high-fat feeding. J Appl Physiol. 2016;121:687-698. doi:10.1152/ japplphysiol.00456.2016

83. Salminen A, Kauppinen A, Kaarniranta K. FGF21 activates AMPK signaling: impact on metabolic regulation and the aging process. J Mol Med. 2017;95:123-131. doi:10.1007/s00109-016$1477-1$
84. Kruse R, Vienberg SG, Vind BF, Andersen B, Hojlund K. Effects of insulin and exercise training on FGF21, its receptors and target genes in obesity and type 2 diabetes. Diabetologia. 2017;60:2042-2051. doi:10.1007/s00125-017-4373-5

85. Liu SQ, Roberts D, Kharitonenkov A, et al. Endocrine protection of ischemic myocardium by FGF21 from the liver and adipose tissue. Sci Rep. 2013;3:2767. doi:10.1038/srep02767

86. Cong WT, Ling J, Tian HS, et al. Proteomic study on the protective mechanism of fibroblast growth factor 21 to ischemia-reperfusion injury. Can $J$ Physiol Pharmacol. 2013;91:973-984. doi:10.1139/cjpp-2012-0441

87. De Sousa-coelho AL, Marrero PF, Haro D. Activating transcription factor 4-dependent induction of FGF21 during amino acid deprivation. Biochem J. 2012;443:165-171. doi:10.1042/ BJ20111748

88. Zhang $\mathrm{C}$, Huang $\mathrm{Z}, \mathrm{Gu} \mathrm{J}$, et al. Fibroblast growth factor 21 protects the heart from apoptosis in a diabetic mouse model via extracellular signal-regulated kinase 1/2-dependent signalling pathway. Diabetologia. 2015;58:1937-1948. doi:10.1007/ s00125-015-3630-8

89. Planavila A, Redondo-Angulo I, Ribas F, et al. Fibroblast growth factor 21 protects the heart from oxidative stress. Cardiovasc Res. 2015;106:19-31.

90. Aubert G, Vega RB, Kelly DP. Perturbations in the gene regulatory pathways controlling mitochondrial energy production in the failing heart. Biochim Biophys Acta. 2013;1833:840-847. doi:10.1016/j.bbamcr.2012.08.015

91. Hsuchou H, Pan W, Kastin AJ. The fasting polypeptide FGF21 can enter brain from blood. Peptides. 2007;28:2382-2386. doi:10.1016/j.peptides.2007.10.007

92. Tan BK, Hallschmid M, Adya R, Kern W, Lehnert H, Randeva HS. Fibroblast growth factor 21 (FGF21) in human cerebrospinal fluid: relationship with plasma FGF21 and body adiposity. Diabetes. 2011;60:2758-2762. doi:10.2337/db11-0672

93. Owen BM, Ding X, Morgan DA, et al. FGF21 acts centrally to induce sympathetic nerve activity, energy expenditure, and weight loss. Cell metab. 2014;20:670-677. doi:10.1016/j.cmet.2014.07.012

94. Owen BM, Bookout AL, Ding X, et al. FGF21 contributes to neuroendocrine control of female reproduction. Nat Med. 2013;19:1153-1156. doi:10.1038/nm.3250

95. Shahror RA, Linares GR, Wang Y, et al. Transplantation of mesenchymal stem cells overexpressing fibroblast growth factor 21 facilitates cognitive recovery and enhances neurogenesis in a mouse model of traumatic brain injury. $J$ Neurotrauma. 2020;37:14-26. doi:10.1089/neu.2019.6422

96. Sa-Nguanmoo P, Tanajak P, Kerdphoo S, et al. FGF21 improves cognition by restored synaptic plasticity, dendritic spine density, brain mitochondrial function and cell apoptosis in obese-insulin resistant male rats. Horm Behav. 2016;85:86-95. doi:10.1016/j. yhbeh.2016.08.006

97. Sun Y, Wang Y, Chen ST, et al. Modulation of the astrocyte-neuron lactate shuttle system contributes to neuroprotective action of fibroblast growth factor 21. THERANOSTICS. 2020;10:8430-8445. doi:10.7150/thno.44370

98. Taliyan R, Chandran SK, Kakoty V. Therapeutic approaches to Alzheimer's type of dementia: a focus on FGF21 mediated neuroprotection. Curr Pharm Des. 2019;25:2555-2568. doi:10.2174/ 1381612825666190716101411

99. Jiang Y, Lin L, Liu N, et al. FGF21 protects against aggravated blood-brain barrier disruption after ischemic focal stroke in diabetic $\mathrm{db} / \mathrm{db}$ male mice via cerebrovascular PPARgamma activation. Int J Mol Sci. 2020;21:824.

100. Chen J, Hu J, Liu H, et al. FGF21 protects the blood-brain barrier by upregulating PPARgamma via FGFR1/beta-klotho after traumatic brain injury. $J$ Neurotrauma. 2018;35:2091-2103. doi:10.1089/neu.2017.5271 
101. Wang D, Liu F, Zhu L, et al. FGF21 alleviates neuroinflammation following ischemic stroke by modulating the temporal and spatial dynamics of microglia/macrophages. $J$ Neuroinflammation. 2020;17:257. doi:10.1186/s12974-020-01921-2

102. Xiao Y, Liu L, Xu A, et al. Serum fibroblast growth factor 21 levels are related to subclinical atherosclerosis in patients with type 2 diabetes. Cardiovasc Diabetol. 2015;14:72. doi:10.1186/ s12933-015-0229-9

103. Rusu CC, Racasan S, Kacso IM, et al. The metabolic hormone FGF21 is associated with endothelial dysfunction in hemodialysis patients. Int Urol Nephrol. 2017;49:517-523. doi:10.1007/ s11255-016-1474-x

104. Semba RD, Crasto C, Strait J, Sun K, Schaumberg DA, Ferrucci L. Elevated serum fibroblast growth factor 21 is associated with hypertension in community-dwelling adults. J Hum Hypertens. 2013;27:397-399. doi:10.1038/jhh.2012.52

105. Eto K, Tumenbayar B, Nagashima S, et al. Distinct association of serum FGF21 or adiponectin levels with clinical parameters in patients with type 2 diabetes. Diabetes Res Clin Pract. 2010;89:52-57. doi:10.1016/j.diabres.2010.03.019

106. Flier JS. Hormone resistance in diabetes and obesity: insulin, leptin, and FGF21. Yale J Biol Med. 2012;85:405-414.

107. Li X, Fan X, Ren F, et al. Serum FGF21 levels are increased in newly diagnosed type 2 diabetes with nonalcoholic fatty liver disease and associated with hsCRP levels independently. Diabetes Res Clin Pract. 2011;93:10-16. doi:10.1016/j. diabres.2011.02.034

108. Xiao Y, Xu A, Law LS, et al. Distinct changes in serum fibroblast growth factor 21 levels in different subtypes of diabetes. J Clin Endocrinol Metab. 2012;97:E54-8. doi:10.1210/jc.2011-1930

109. Tillman EJ, Rolph T. FGF21: an emerging therapeutic target for non-alcoholic steatohepatitis and related metabolic diseases. Front Endocrinol. 2020;11:601290.

110. Li H, Fang Q, Gao F, et al. Fibroblast growth factor 21 levels are increased in nonalcoholic fatty liver disease patients and are correlated with hepatic triglyceride. $J$ Hepatol. 2010;53:934-940. doi:10.1016/j.jhep.2010.05.018
111. Suomalainen A, Elo JM, Pietilainen KH, et al. FGF-21 as a biomarker for muscle-manifesting mitochondrial respiratory chain deficiencies: a diagnostic study. Lancet Neurol. 2011;10:806-818. doi:10.1016/S1474-4422(11)70155-7

112. Cheng P, Zhang F, Yu L, et al. Physiological and pharmacological roles of FGF21 in cardiovascular diseases. $J$ Diabetes Res. 2016;2016:1540267. doi:10.1155/2016/1540267

113. Davis GR, Deville T, Guillory J, Bellar D, Nelson AG. Relationship between family history of type 2 diabetes and serum FGF21. Eur J Clin Invest. 2017;47:853-859. doi:10.1111/ eci. 12835

114. Tanajak P, Sa-Nguanmoo P, Apaijai N, et al. Comparisons of cardioprotective efficacy between fibroblast growth factor 21 and dipeptidyl peptidase-4 inhibitor in prediabetic rats. Cardiovasc Ther. 2017;35:e12263. doi:10.1111/1755-5922.12263

115. Itoh N, Ohta H, Nakayama Y, Konishi M. Roles of FGF signals in heart development, health, and disease. Front Cell Dev Biol. 2016;4:110.

116. Kim WJ, Kim SS, Lee HC, et al. Association between serum fibroblast growth factor 21 and coronary artery disease in patients with type 2 diabetes. J Korean Med Sci. 2015;30:586-590. doi:10.3346/jkms.2015.30.5.586

117. Shen Y, Ma X, Zhou J, et al. Additive relationship between serum fibroblast growth factor 21 level and coronary artery disease. Cardiovasc Diabetol. 2013;12:124. doi:10.1186/1475-2840-12124

118. Hale C, Chen MM, Stanislaus S, et al. Lack of overt FGF21 resistance in two mouse models of obesity and insulin resistance. Endocrinology. 2012;153:69-80. doi:10.1210/ en.2010-1262

119. Kharitonenkov A, Dunbar JD, Bina HA, et al. FGF-21/FGF-21 receptor interaction and activation is determined by betaKlotho. J Cell Physiol. 2008;215:1-7. doi:10.1002/jcp.21357

\section{Publish your work in this journal}

Diabetes, Metabolic Syndrome and Obesity: Targets and Therapy is an international, peer-reviewed open-access journal committed to the rapid publication of the latest laboratory and clinical findings in the fields of diabetes, metabolic syndrome and obesity research. Original research, review, case reports, hypothesis formation, expert opinion and commentaries are all considered for publication. The manuscript management system is completely online and includes a very quick and fair peer-review system, which is all easy to use. Visit http://www.dovepress.com/testimonials.php to read real quotes from published authors. 